\title{
DIGITAL STORYTELLING IN AFRICA
}

\author{
Gary Marsden ${ }^{1)}$, Ilda Ladeira ${ }^{1)}$, Thomas Reitmaier ${ }^{1)}$, \\ Nicola J. Bidwell ${ }^{2)}$, Edwin Blake ${ }^{1)}$
}

\footnotetext{
1) Department of Computer Science, University of Cape Town, South Africa, gaz@acm.org, http://www.cs.uct.ac.za/ ${ }^{2)}$ Meraka Institute of the CSIR, Pretoria, South Africa, nic.bidwell@gmail.com, http://www.meraka.org.za/
}

\begin{abstract}
In this paper we examine how digital technology can be used to inspire, record and present oral stories in an African context. In particular we explore how to create technologies that are sympathetic to the cultures of the storytellers, both in the capture of stories and their retelling. Specifically, we look at: inspiring stories in District Six in Cape Town; capturing digital stories from users with low literacy levels and using virtual reality to retell indigenous and personal experience narratives.
\end{abstract}

Keywords: HCI, Storytelling, ICT4D, Africa, Museum.

\section{INTRODUCTION}

Technology and storytelling may not seem like an appropriate match, especially in a continent such as Africa where technology penetration is relatively low. However, in our research we have found that interactive digital technology is ideally suited to capture oral stories in a way that is not possible through non-interactive media, such as text or video. Beyond simply capturing stories, we also explore how digital technology can be used to store and curate existing stories.

In the rest of the paper we describe bodies of work aimed at the capture and presentation of stories on digital systems. We first describe a mobile system that allows non-literate users to plan and record their own digital stories. We then look at the use of virtual reality for preserving indigenous folklore.

\section{DIGITAL STORIES}

The late Dana Atchley developed "digital storytelling" in California in the early to mid-1990s with the idea of putting "the universal human delight in narrative and self-expression into the hands of everyone" [1]. Atchley developed an exportable workshop in which ordinary people are taught how to produce their own digital stories. Essentially, a digital story is a collection of still images joined and overlaid with a voice narrative. This exportable format has led to the impressive spread of digital storytelling across the world. However, digital storytelling has not spread evenly around the world, for it is practiced less in Asia, Africa, and South America [1]. This comes to little surprise to us, as "exporting" a technology, method, or approach into Africa has often fallen short or failed [2]. While it is easy to attribute this uneven spread to the low literacy and computer literacy rates found in Africa, perhaps the more profound reason for digital storytelling's failure in Africa is that the workshop approach is built upon Western storytelling practices and is, thus, insensitive to the rich oral traditions found all over the continent [3].

However, we believe that digital storytelling could play a role in rural African communities, as there appears to be a high degree of compatibility between the rich oral storytelling heritage of those communities and the audio narrative of digital stories. In our research we aim to address the imbalance mentioned earlier by not only creating an easy-to-use mobile digital storytelling system but, more importantly, allowing people living in rural African communities to shape their own form of digital storytelling compatible with their ways of doing and saying [4].

The mobile phone has had a tremendous impact on the livelihoods and lives of people everywhere, who are using the device for "both productive and personal uses through their daily routine" [5]. From our first hand experiences of living in and visiting rural communities in South Africa and Kenya, we observed the increasing availability and usage of the mobile in rural areas. Because community members are familiar with mobile phones, albeit mostly with basic Nokia 1100 type phones, we targeted it as our 


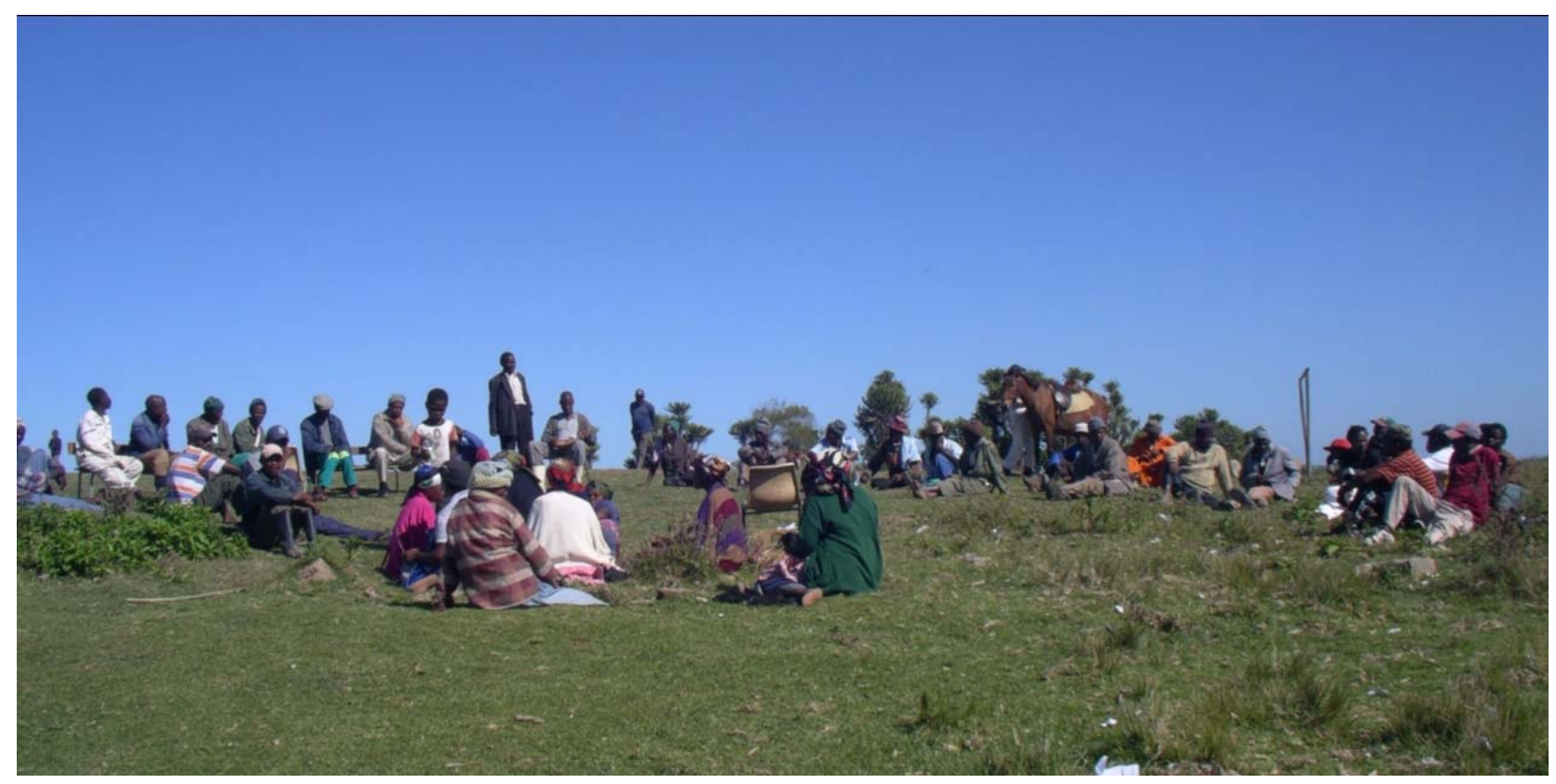

Fig. 1 - A Village Meeting in Lower Ndungunyeni

storytelling device. The social nature of the mobile along with its communication potential and different media input and output capabilities make it an ideal digital storytelling platform, where people can integrate and playback visual and audio media.

\subsection{PREVIOUS WORK}

We had previously been involved with a technology-inspired and user-focused project aimed at combining the two technologies of digital storytelling and mobile phones. For the project we iteratively designed and evaluated, with university students, two mobile digital storytelling interfaces. With the story-driven interface users record a narrative first and then add in photos; while, with the photo-driven interface users add photos to a storyline and then annotate these by recording audio. Our usage scenario was one in which a user combines a set of three photos with an audio recording of reading from a pre-scripted story. We iteratively refined these two interfaces by creating and evaluating with university students a paper, a PowerPoint, and two high-fidelity Flash Lite prototypes. To explore a more elaborate interaction scenario, where users construct their own story, rather than read a scripted one, we also developed a further application prototype. In this prototype, which was informed by usability outcomes from previous prototypes, users could record and select their own audio and photos. However, we decided against evaluating and refining this prototype outside of the urban setting where it was designed, as we noticed vastly different storytelling traditions in the rural communities we visited.

\subsection{ETHNOGRAPHIC LENS AND DESIGN WORKSHOP}

Our ethnographic perspective on storytelling is informed by data gathered independently of developing initial prototypes and is situated in Lower Ndungunyeni in the Wild Coast of South Africa's Eastern Cape (see Figure 1) [4]. Insights on storytelling, oral and digital communication emerged over 18 months as we formed relationships, interpreted priorities, discovered design opportunities in the ad-hoc details of daily life, and undertook socio-technical experiments [4]. The insights we gained in-situ, together with our previous experiences on mobile digital storytelling, led us to develop a digital storytelling design workshop in the village Tschani. The aim of our workshop was to allow users to shape the design of a mobile digital storytelling system suited to their community; so, we focused all activities around a pair of phones, where one phone functioned as a voice recorder/player and the other as a camera and photo viewer. We ran the workshop at a local NPO (Non-profit Organisation) and recruited six participants via the NPO. The rudimentary nature of the phone-pair provided participants with enough ambiguity to explore different ways of incorporating visual and audio media in storytelling activities. We asked our participants to form three groups and spread the workshop over two consecutive afternoons, so participants could engage others and gather story ideas and material in a more natural setting. After explaining and practicing the use of the phone-pair's voice recorder, camera, and gallery 
during the first afternoon, the groups recorded their digital stories on the second afternoon in a variety of different ways. By reviewing the video and notes we took during the workshop, we were able to design a mobile digital storytelling prototype better suited to the needs of rural, oral users.

\subsection{CURRENT PROTOTYPE}

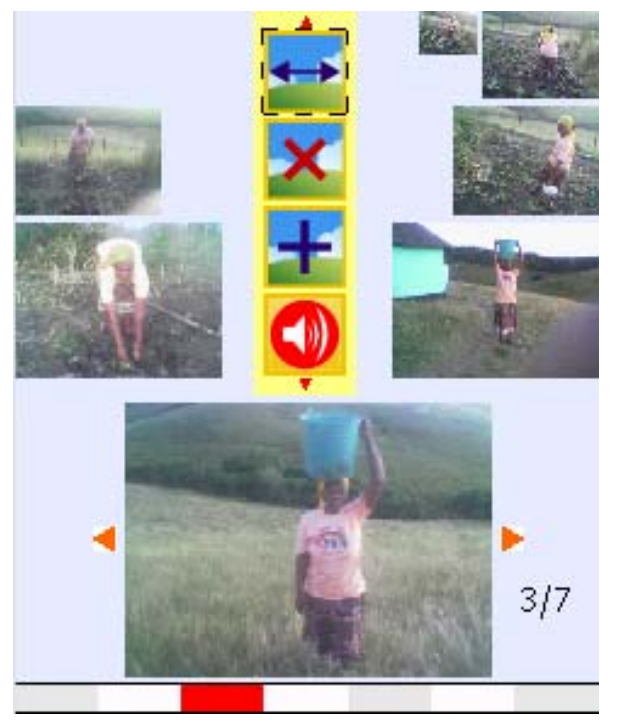

Fig. 2 - The interface to the mobile digital story application

The biggest improvement of our current prototype over our initial prototypes is a flexible and text-free interface, as shown in Figure 2. Its flexibility allows users to create stories in a variety of ways without imposing a constraining storytelling style. Users can record audio first and later annotate it with pictures. Alternatively, they can select pictures first and then record a voice-over; or they can use a hybrid approach. At any time, they can add, move, or remove pictures and append or overwrite audio. All necessary functions can be accessed via a scrollable vertical toolbar of icons, which allows even illiterate users to create digital stories in their mother tongue. With this application we have created a rich means for rural users to tell their stories in a way that is natural to them. By studying its use we can leverage the application as a tool to further situate digital storytelling in rural African communities.

\section{PRESERVING STORIES}

Even once stories are collected, presenting them in way that is faithful to their original contexts poses complex problems. Often, the person listening to the story will be in a physical environment completely different to the one in which the story was recorded. Furthermore, the listener may lack the cultural experience required to interpret the story. Finally, in the case of oral storytelling, the listener is not likely to be able to interact with the storyteller.

We looked to virtual reality (VR) to overcome these some of these barriers particularly in relation to oral storytelling. Many African cultures may be described as predominantly oral; knowledge is preserved by telling rather than writing, and storytelling performances are an important social and culture-defining practice. Stories ranging from indigenous folktales to stories about relatively recent historical periods, such Apartheid-era narratives, may be preserved as text or video. However these media lack the original live oral dimension of these stories. Virtual reality has been used in cultural heritage work to reconstruct historical sites and artifacts that that no longer exist. We endeavoured to extend this use to recreate storytelling experiences which may no longer possible in real life.

\subsection{SAN STORIES}

Cultural heritage preservation work has recently begun to include efforts towards preserving "intangible" cultural heritage (ICH). This definition includes things such as rituals, skills, expressions, which are typically preserved orally. We created two virtual environments (VEs) aimed at recreating an intangible cultural experience from San culture.

The San (also known as Bushmen) are an indigenous hunter-gatherer people of southern Africa, whose culture dates back over 20000 years [6]. Since the start of colonization in the late 1400's, San populations decreased dramatically and their lifestyle has become almost extinct. Among the most valuable records of San culture are their famous rock art sites [7]. The San also have a rich oral culture and a tradition of storytelling and orality is all pervasive and defining of San culture [8]. Today there are very few San living the traditional huntergatherer lifestyle, and none from the Southern San. The Southern San or |Xam culture is no longer alive, but in the late nineteenth century Wilhelm Bleek, Lucy Lloyd and Dorothea Bleek collected a meticulous archive of |Xam folklore from February 1871 to October 1873 [6]. The sources for the stories were \|Kabbo and his son-in-law han kkass'o. |Kabbo's (whose name means "dream"). This elder of his community was imprisoned by the colonial authorities and expressed the wish that his stories be known by way of books.

Today the Bleek and Lloyd archive, housed at the University Cape Town, is one of the most extensive archives of San folklore and have been digitised to DVD [9]. Records such as reflect the San's extensive oral storytelling tradition and lend invaluable insight into their culture.

We created two virtual environments aimed at recreating the experience of San oral storytelling. In 
the first, we explored how the sounds and visuals might enhance a user's experience of the story presented. The virtual environment was very simple and featured a cave at night time. Inside the cave there is a fire, surrounded by three figures, conceptualized as San hunters setting up camp for the night. One of the hunters tells a traditional folktale to the other two hunters and the user. Initially the user is placed just outside the cave; upon entering they are invited to sit and listen to a story about Kaggen the Mantis, the classical trickster figure of San mythology.

The story was taken from the Bleek and Lloyd collection mentioned earlier and tells of a prank played by Kaggen on a village. The story participants are animals since San folklore typically takes place in a time where animal and human forms are interchangeable.

Our VE, shown in Figure 3, was used in a study to test the effects of including ambient audio elements and visual elements which related to the story being told.
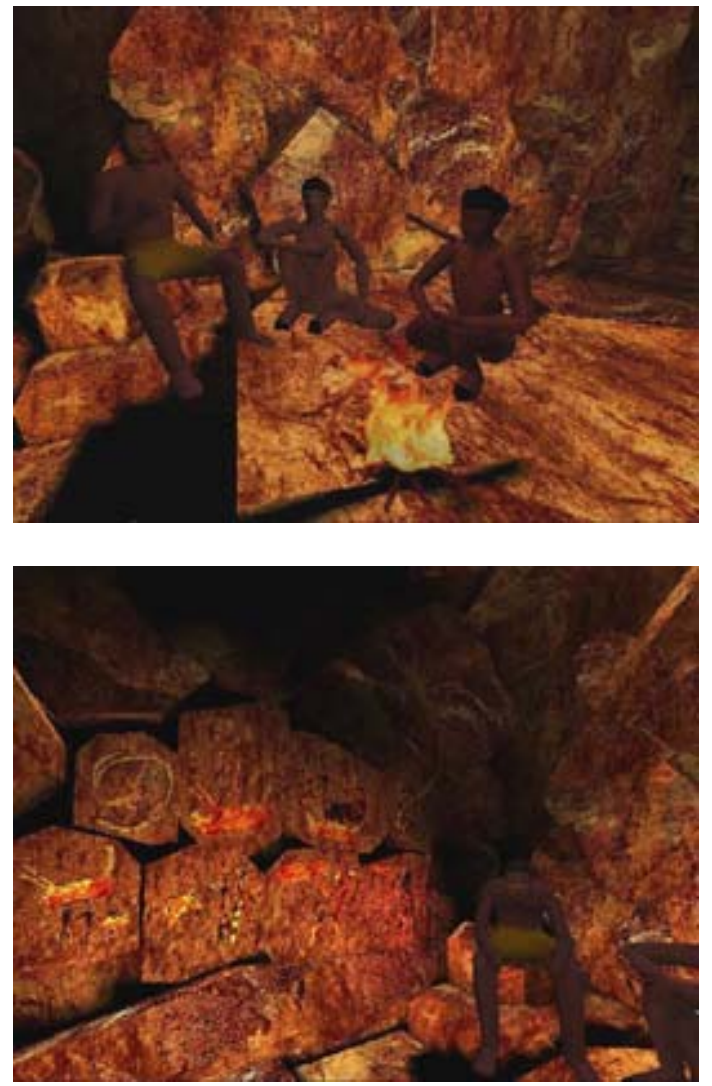

Fig. 3 - Screenshots from a virtual environment where a user could experience fireside San storytelling among a group of hunters. The top shows the three hunter avatars sitting around a fire. The lower figure shows the rock art depicting events from the story told, the addition of these visuals was found to increase presence, story involvement and, when used in combination with ambient audio sounds, story enjoyment
Ambient audio element took the form of sounds such as fire crackling and crickets chirping. Meanwhile story-related visual were implemented as San rock paintings depicting story events on the cave wall nearest to the storyteller. As the storyteller avatar told the story, a subtle light was used to illuminate the painting relating the part of the story being told. Using a retrospective questionnaire we measured study participants' presence, i.e. the extent to which they experienced the VE as a real place and their level of involvement in and enjoyment of the story. In a study run with 77 university students, we found the addition of ambient audio significantly increased presence $(\mathrm{F}=138.8, \mathrm{p}<0.002)$ and enjoyment of the story $(\mathrm{F}=4.01, \mathrm{p}<0.05)$. The storyrelated visuals increased involvement in the story $(\mathrm{F}=9.49, \mathrm{p}<0.003)$ and the combination of visual and ambient audio significantly enhanced story enjoyment $(\mathrm{F}=11.11, \mathrm{p}<0.002)$ [11].

The second investigation of using VR to recreate traditional San storytelling was guided by the difficulty of creating compelling VEs. Creating a good VE requires a great deal of effort and a range of skills such as graphics programming, soundtrack recording and $3 \mathrm{D}$ modeling and animation. This, firstly, prompted us to explore whether this investment was worthwhile by critically evaluating VR's effectiveness as a storytelling medium. To this end, we decided to compare a storytelling VE to a story text, a more common medium for presenting indigenous stories. At present, San folklore is almost exclusively accessible through texts such as the Bleek and Lloyd collection. While we had previously assumed that reimagining textual stories in a VE would be more engaging than reading, we decided to test this explicitly. We hypothesised that presenting a San story via a storyteller avatar in a VE would capture aspects of its original, performative spirit and provide a richer cultural contextualisation.

Secondly, we set out to explore a novel way to boost the effectiveness of using VR for cultural heritage storytelling. In particular, we were concerned that San story content might be too culturally remote to most users and, thus, fail to engage them. To address this we explored the use of priming, a technique where users are exposed to material related to a VE's content before experiencing the VE. This is intended to place users in a frame mind that is receptive to the VE's content. [Nunez 2003] Priming material is usually related to the VE content, and we wished to examine whether it would be useful for making potentially culturally remote content more familiar and engaging. We decided to test the use of priming in the form of a culturally familiar introductory VE which foreshadowed the San VE content. This required that 
we choose a cultural theme that was likely to be familiar to most users. To this end, we chose hiphop, since it is a well-known contemporary subculture with a distinctive and easily recognisable style. However, any other well-known, contemporary subculture might have been appropriate. We felt that hip-hop fit the bill for our work because it shares some key similarities with San culture. Key aspects of San culture is their storytelling, rock art, music and dance traditions [7]. These may be seen to correspond to the key aspects of hip-hop culture: rapping, graffiti, dj-ing and break dancing, respectively [12]. Among these, the link between rapping and oral storytelling particularly struck us. Rapping is a musical style where lyrics are spoken or chanted over music, which is often heavily sampled [13]. Rap has been described as a hybrid cultural expression which incorporates a great number of expressive systems, including African storytelling and "street-smart moral fables" [13].

To aid us in evaluating the storytelling effectiveness of VR and our introductory VE technique, we carefully considered our measures for storytelling success. We considered what might characterise a successful cultural storytelling experience beyond the presence, story involvement and story enjoyment measures we had used previously. We conceptualised an effective storytelling experience as one where the story is understood and enjoyed; we also felt that it should foster an interest in the culture concerned and make culturally remote stories accessible to most audiences.

The San VE created for this study was much more detailed than our first. We paid particular attention to ensuring that that the story content, avatars and physical environment were authentic. The VE was, again, set in a cave at dusk, with a San gathering sitting around a fire. The cave was modelled on the shallow caves found in the Cederberg mountains, a region once inhabited by the San and one of the richest regions of San rock art [7]. Digital images of Cederberg caves were used to texture the virtual cave. In accordance with findings in our previous work, we included ambient audio and textured the cave with photographs of rock art which related to the story that would be told in the VE. [6] The San gathering consisted of an adult man, two children and an elder woman, who acts as the storyteller.

In order to ensure accurate appearance, in terms of clothing and physique, for these avatars we enlisted the help of an artist and an archaeologist expert on the San. Photographs of the San were used by the artist to create detailed reference sketches of the four San characters. Before modelling, these sketches were reviewed by the archaeologist expert. On the first review, significant corrections were made to the sketches and once they were found to be accurate, modelling of the avatars began. Once again, we consulted the archaeologist for guidance with this adaption before recording the narration audio.

In the $\mathrm{VE}$, the user begins some distance from the cave; this view is shown in Figure 4. As the user moves toward the gathering, the San man stands up and looks in their direction. During this time a simple African music track plays in the background along with ambient sounds such as wind blowing and crickets chirping. When the user has almost reached the gathering, the music fades and the San man invites the user to move closer to the gathering. However, if the user takes longer than ten seconds to walk toward the gathering, the San man also offers the following encouragement. Figure 5 shows the San man inviting the user to come closer after which the elder storyteller, shown in Figure 6, says:

"I was just about to tell a story, the one about how the Mantis made the Eland, please sit down with us and listen."

The storyteller then begins to tell the story, during this time the user is free to explore the cave area and view the fire, rock paintings and other objects typical of a San living area such a grinding stone and a bag and quiver hanging from wooden pegs on the cave wall. These objects were created and placed in accordance with San reference material and the advice of our archaeologist consultant. The San gathering react during the storytelling by exclaiming, gesturing and moving their heads; a screenshot of this is shown in Figure 7. The storyteller avatar is also animated throughout the story narration. The voice of the storyteller was provided by a local voice-over actress. During this voice recording her movements and hand gestures were video-taped and, subsequently, rotoscoped for the storyteller avatar's animations.

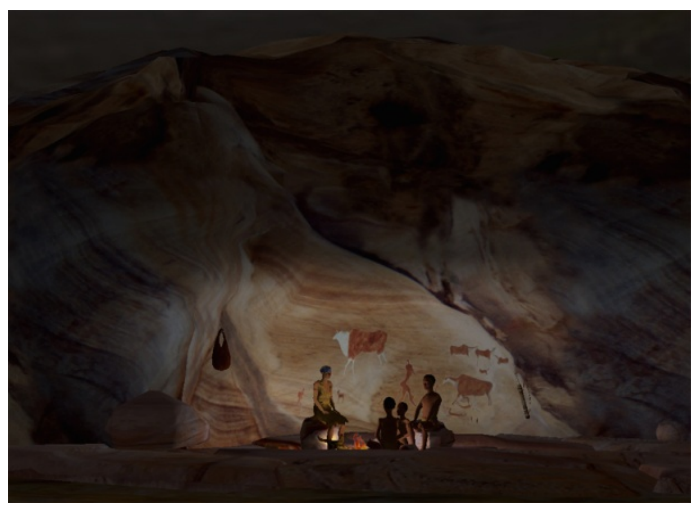

Fig. 4 - Screenshot showing the user's starting point in the San virtual storytelling environment. In the distance a San gathering sitting around a fire in a shallow cave are visible 


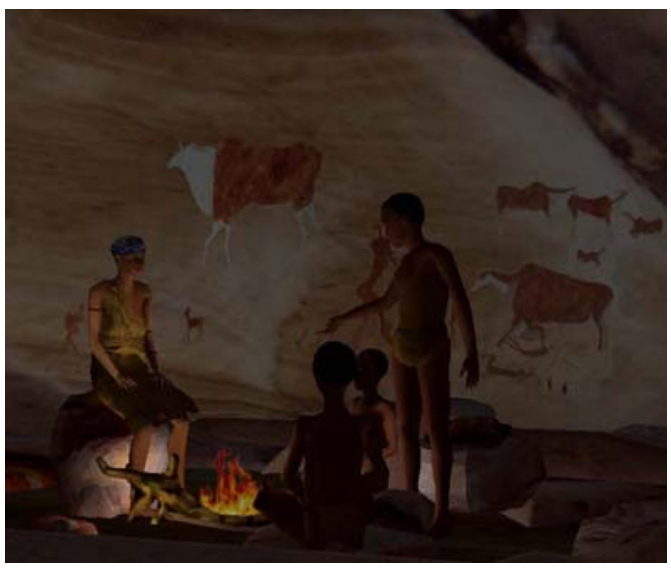

Fig. 5 - Screenshot from the San virtual storytelling environment which shows the San man from the gathering standing to greet and invite the user the group

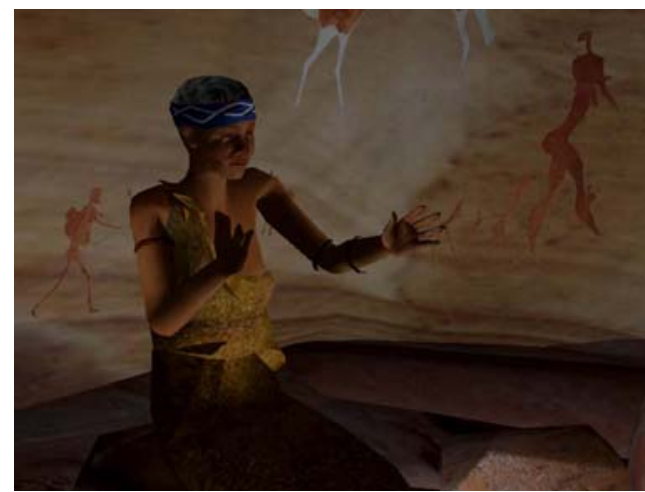

Fig. 6 - Screenshot of the storyteller avatar, an elder San women, tells an indigenous story How Kaggen the Mantis created the Eland and the Moon to a San group around the fire and the user

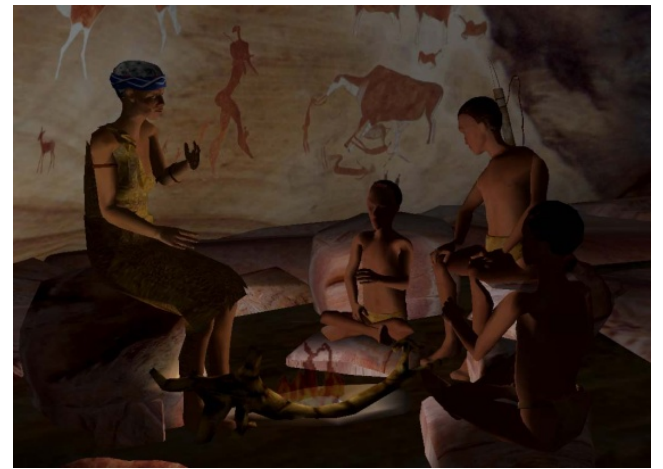

Fig. 7 - Screenshot from the San virtual storytelling nvironment taken during the story narration. The storyteller avatar is animated throughout the narration and the San gathering reacts through animations and audible exclamations and gasps

The priming introduction VE presents the user with an urban environment containing a hip-hop avatar with a radio. The avatar is rapping about the San people and the story that will be told in the San VE. Again, the recorded actions of a real-life actor, in this case a hip-hop musician, were rotoscoped to animate the hip-hop avatar. Behind the hip-hop avatar we created a number of graffiti covered walls. One of the walls contains a door with the word "San" as graffiti on it. When the user starts in the VE the hip-hop avatar is visible and raps the following about the San in a loop:

"You gotta understand the San,

Indigenous peeps of da motherland.

Making rock art, workin' real hard.

Huntin', yo peeps be frontin'.

We be jammin', dancin' rhymin'

Making mad beats now for keeps

I hopes you like dis story about Him

Kagg'n da Mantis speaks

Don't despise this gifted, thrifted, swifted and mighty

Creator, da maker of da Hartebeest, da moon,

Soon you gon hear how he made the E-land too

So listen to me man as I usher you into the world of the San.

Let's jam!'

As the user walks toward the avatar, he stops rapping and speaks to the user telling about the San and their storytelling tradition:

"Yo! Did you know that way back in the day, the

San used to live right here? Right through that door, you can hear a real San story!"

This monologue, along with the earlier rap, serves as preparation for the main VE's content. He then directs the user to the "San" door, which opens to reveal the San VE. Both VEs were implemented using an in-house VE authoring tool called VR Direct, a scripting engine and user interface built on top of Gamebryo, a commercial games engine.

We conducted two studies using these VE's with different samples: (1) 44 high-school children aged 15 to 17 and (2) 98 undergraduate university students. We made two main story experience comparisons. Firstly, between participants experiencing the story in the VE with those reading the story as text and, secondly, between those who experienced the San VE with and without the priming VE. Story experience was measured using retrospective questionnaires for the following dependant variables:

- Comprehension: a number of tests, drawn from educational research, were used to test understanding and recall of the San story.

- Interest: interest in and desire to find out about San culture and folklore.

- Enjoyment of the story.

- Boredom during the story experience.

- Confusion about the story content.

- Presence in the San VE 
For our second study, we added the following dependant variables:

- Attention paid to the San story.

- Perceived strangeness of the San story.

We also measured Hip-hop Interest, using a number of questionnaire items in which participant's rated their affinity for hip-hop culture. This was done so that we could factor this into our analysis of the effect of our priming VE.

\subsection{STORY EXPERIENCE:}

The correlations among our dependant variables strongly suggested that our conceptualisation of story experience was sound:

- Comprehension, interest and enjoyment correlated positively.

- Boredom and confusion correlated positively.

- Comprehension correlated negatively with both boredom and confusion.

- Interest and enjoyment both correlated negatively with boredom and confusion.

- Attention correlated positively with comprehension, interest and enjoyment and negatively with boredom and confusion.

- Strangeness correlated negatively with interest and enjoyment and positively with boredom and confusion.

\subsection{VIRTUAL REALITY VS. TEXT:}

Among the high-school students, we found that comprehension was significantly higher for those reading the story $(\mathrm{F}=23.54 ; \mathrm{p}<0.0001)$. However, results for the other story experience variables pointed strongly towards a more favourable experience with VR: participants experiencing the VE showed significantly higher enjoyment $(\mathrm{F}=17.67, \quad \mathrm{p}<0.001), \quad$ less boredom $(\mathrm{F}=7.44$, $\mathrm{p}<0.009)$, less confusion $(\mathrm{F}=15.54 ; \mathrm{p}<0.001)$ and greater interest in San culture $(\mathrm{F}=5.639 ; \mathrm{p}<0.02)$ [Ladeira, 2004]. However, in the university student sample, we found fewer significant differences. Comprehension was still significantly higher for the text story $(\mathrm{H}=33.07, \mathrm{p}<0.00001)$ while confusion was lower $(\mathrm{F}=5.10, \mathrm{p}<0.026)$. Additionally, we found that attention to the story was significantly lower in the storytelling $\mathrm{VE}(\mathrm{F}=8.13, \mathrm{p}<0.005)$ while perceived strangeness of the story was significantly lower $(\mathrm{H}=6.04, \mathrm{p}<0.014)$. We posit that the lack of differences for enjoyment, boredom or interest in our second study reflects a difference in reading attitudes and skills in our two samples. For instance, the high-school students who read the story reported strikingly very low levels of engagement with the story, despite scoring high on comprehension. Meanwhile, the university student sample exhibited similar levels of engagement for the textual and virtual story media. We believe that the university student sample represents a highly selected sample with above average reading skills and a greater likelihood to responding positively to reading. An analysis combining the high-school and university samples showed an, overall, more favourable experience in the storytelling VE where there was significantly greater enjoyment $(\mathrm{F}=8.47, \mathrm{p}<0.004)$ and interest $(\mathrm{F}=4.77, \mathrm{p}<0.031)$ and significantly lower boredom $(\mathrm{F}=4.23, \mathrm{p}<0.042)$.

However, our results were not clear-cut, suggesting that neither text nor VR proved to be the "winning" story medium. In fact, there seemed to be trade-off between conveying the story content and providing a fun experience which generates cultural interest. We could classify comprehension as a variable relating to conveying content and the interest, enjoyment, confusion and boredom as variables relating to the engaging experience. Our results suggested that content and experience might not come hand-in-hand, leaving us with a choice: which of these two is the more important goal for cultural storytelling? Say story content is understood and retained, but the experience is not engaging and does not necessarily prompt further exploration of the cultural context. Is such a once-off story experience satisfactory? While our VR users did not grasp and retain the story content as fully as text readers, they had, what we would term, a more effective story experience. Furthermore, a result we found striking was the apparent disconnection between comprehension and confusion. Those reading the story showed better comprehension and attention to the story but greater confusion (for the high-school sample) and greater perceived strangeness of the story (for the university sample). This suggested that, despite better retention of story content, those reading the story found it confusing or strange. Various elements of the story's style, such as vernacular rhyming and repetition patterns and story events, were likely to be unfamiliar to our sample. However, the marked difference in confusion between the VR and text conditions suggests that placing the story in context and hearing it told out loud in the VE made the story more accessible. We believe these results point towards VR's potential for bringing indigenous oral stories, such as those of the San, to life.

\subsection{THE USE OF A PRIMING VIRTUAL ENVIRONMENT:}

While the use of the hip-hop themed priming VE didn't have an effect on overall story experience, we 
did note two interesting effects with each sample. For the high-school sample, we found that Hip-hop Interest was a significant predictor of story enjoyment. So those who indicated a high affinity for hip-hop and experienced the priming VE enjoyed the San story significantly more than those who did not see the priming VE. This suggests that the priming VE only offered a benefit where it tapped in the participants' pre-existing interests.

Among the university student sample, those who identified hip-hop as a favourite music genre and experienced the priming VE $(n=6)$ reported significantly greater presence in the San VE $(\mathrm{F}=5.31, \mathrm{p}<0.029)$ than those who chose other genres $(n=24)$. Furthermore, for those who did not experience the priming VE, no effect was noted for those who chose hip-hop as a favourite $(n=5)$ vs. other genres $(n=23)$. This showed a definite interaction between the priming VE and participant's preference for hip-hop music. While this result is different to that in the high school sample, we were, again, seeing a story experience benefit arise where priming material tapped into preexisting interests [Ladeira, 2005]. We believe that, for participants with a liking for hip-hop, the introductory VE acted as a hook, piquing their interest at the outset of their virtual experience and increasing their overall engagement in the San storytelling VE. However this is an area that we would like to investigate further.

The VE created for this study was ultimately put on temporary exhibit at the IZIKO South African Museum in Cape Town. The VE was projected onto a wall which visitors could navigate using a joystick.

\section{CONCLUSION}

In all of the research presented, the unifying concept is that of user-centred design [Preece, 2008]. We have not tried to impose a technology from outside Africa into an African situation. Instead, we have worked with individuals, and the cultures they represent, to give them a digital voice and ensure their stories survive in a digital landscape. But the benefit is not only to the communities we work with, by building these systems we have learnt much more about how we ourselves communicate and the value of a good story, regardless of the culture it comes from and the media that is used to convey it.

\section{ACKNOWLEDGEMENTS}

Different parts of this work has been funded by the South African (SA) Innovation Fund, SA National Research Foundation, SA Department of Trade and Industry and the Telkom Centre of Excellence. We are also grateful for the support of IZIKO Museums, Bill Tucker from the University of the Western Cape and Transcape for facilitating our studies. Finally, we would like to thank Chris Robson and Grant James for coding some of the work reported here. We wish to thank Prof John Parkington, our design team and Vera Vukovic (Artist), Dawn Langdown (storyteller), Oswald Msuokwa (music and rapper).

\section{REFERENCES}

[1] Hartley J. and McWilliam K. Computational Power Meets Human Contact. In J. Hartley and K. McWilliam, Story Circle: Digital Storytelling Around the World. WileyBlackwell, Oxford, UK, 2009.

[2] Marsden G., Maunder A. and Parker M. People are people, but technology is not technology. Philosophical transactions. Series A, Mathematical, physical, and engineering sciences, 366, 1881 (2008), pp. 795-804.

[3] Finnegan R.H. The Oral and Beyond: Doing Things with Words in Africa. University of Chicago Press, 2007.

[4] Bidwell N.J., Reitmaier T., Marsden G., and Hansen S. Designing with Mobile Digital Storytelling in Rural Africa. CHI'10, ACM Press (2010).

[5] Donner J. Blurring livelihoods and lives: The social uses of mobile phones and socioeconomic development. Innovations: Technology, Governance, Globalization, 4, 1 (2009), pp. 91-101.

[6] Parkington J. The Mantis, the Eland and the Hunter. Creda Communications, Cape Town, 2002.

[7] Parkington J. Cederberg rock paintings. Creda Communications, Cape Town, 2003.

[8] Guenther M. N//àe ("Talking"): The Oral and Rhetorical Base of San Culture. Journal of Folklore Research, 43 (3) (2006), pp. 241-261.

[9] Skotnes P. Claim to the Country: The archive of Lucy Lloyd and Wilhelm Bleek. Jacana, Cape Town. 2007.

[10] Lloyd L., Bleek W., Bleek D. The Mantis and his Friends: Bushman folklore. Maskew Miller, Cape Town, 1923.

[11] Brown S., Ladeira I., Wintebottom C., Blake E. The effects of mediation in a storytelling virtual environment. In Virtual Storytelling: Using Virtual Reality Technologies for Storytelling (2003), Springer-Verlag. Proc. 2nd International Conference ICVS.

[12] Ards A. Organising the hip-hop generation. That's the joint! The hip-hop studies reader. M. Forman \& M. A. Neal. New York and London, Routledge, 1999, pp. 311-23.

[13] Shusterman R. Challenging conventions in the 
fine art of rap. That's the joint! The hip-hop studies reader. M. Forman \& M. A. Neal. New York and London, Routledge, 1992, pp. 45979.

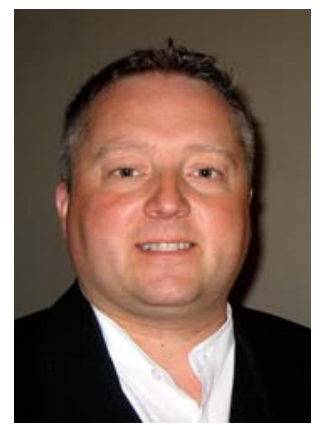

Gary Marsden, is a professor in the Computer Science Department at the University of Cape Town. His research interests are in Mobile Interaction Design and ICT for Development. He has coauthored a book, with Matt Jones titled "Mobile Interaction Design" which was published in 2006. He is currently director of the UCT ICT4D research centre and the Hasso Plattner Research School. He won the 2007 ACM SIGCHI Social Responsiveness award for his research in using mobile technology in the developing world.

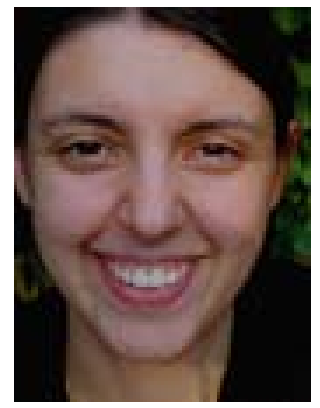

Ilda Ladeira, is a PhD student at the University of Cape Town where she also completed her Masters. She has been involved a number of research projects on preserving stories as cultural heritage using inexpensive virtual reality. She is interested in creating virtual storytelling environments that provide an effective experience of story content. Hence, she has also explored ways to quantitatively measure story experience. She is currently working together with the District Six Museum in Cape Town on creating a virtual environment which presents the personal narratives of people who experienced forced home removals during Apartheid. This work includes studying real-life storytelling though ethnography and creating virtual storytelling agents which simulate realistically interactive and dynamic oral storytelling.

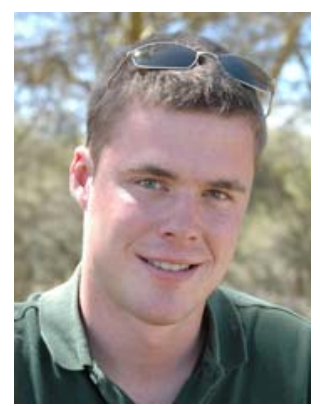

Thomas Reitmaier, is a Masters student at the Centre for ICT4D of the University of Cape Town. Thomas grew up in Washington D.C. before studying Computer Science in Aachen, Germany. Since his parents moved to Kenya in 2004, he has spent a lot of time in Africa. A semester abroad brought him to the University of Cape Town, where he has since transferred to. During his time in Africa he has seen the great impact that mobile phones have had on the continent. This led him to develop an interest in Mobile Interaction Design, which he now passionately pursues.

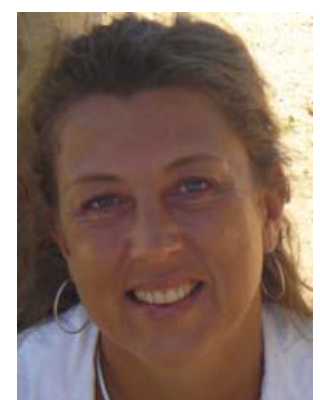

Nicola J. Bidwell, is a researcher at the Meraka Institute and an Honorary researcher at Nelson Mandela University, South Africa. She spent the first few years of life in Africa and has been a Third Culture Kid since. Since 2003 she focused on $\mathrm{HCl}$ for rural contexts and 'Indigenous' cultural logics (e.g. in Aboriginal communities in Australia and villages in South Africa, Namibia and Mozambique). She intends to design for 'envelopment' and most of her peer-reviewed publications relate to designing interactions with mobile devices, information systems and simulated$3 D$ environments that suit the needs of inhabitants of, and visitors to, rural and often impoverished or geographically remote places. Nic gained her PhD in neurophysiology (1992) but then undertook a Masters in IT (2001); has Honours in Biology and Psychology; but, these days, draws on ethnographic and participatory strategies. She has studied, researched and/or lectured at Universities of Stirling, London, Sussex and Cambridge, the Australian National, Queensland, Charles Darwin Universities and at the University of Cape Town. She established the Games Design Programme at James Cook University, Australia, where she was also Deputy Head of the School of IT.

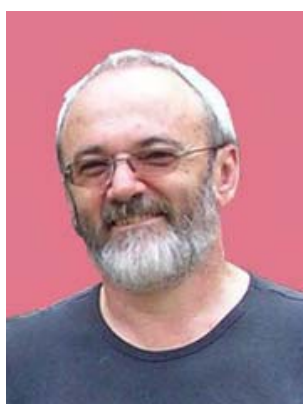

Edwin Blake, is a professor in Computer Science at the University of Cape Town. His work focuses on the relations of people to computers in the context of a developing country.

His schooling and undergraduate education was obtained in South Africa. He read for a PhD in Computer Science at Queen Mary College, London University. He was subsequently senior researcher in the Department of Interactive Systems at the Centre for Mathematics and Computer Science (CWI) in Amsterdam, the Netherlands.

His recent research focus has been on Information and Communications Technology for Development and User Experience as applied to Games and Virtual Environments. His research outputs range from reflections on policy issues to effective interaction design. 